\title{
NEURODESENVOLVIMENTO DE LACTENTES NASCIDOS A TERMO PEQUENOS PARA A IDADE GESTACIONAL NO SEGUNDO MÊS DE VIDA
}

\author{
Maura M. Fukujima Goto', Vanda M. Gimenes Gonçalves², Abimael Aranha Netto³, \\ André Moreno Morcillo ${ }^{3}$, Maria Valeriana Leme de Moura-Ribeiro ${ }^{4}$
}

\begin{abstract}
RESUMO - O objetivo deste estudo foi avaliar e comparar o neurodesenvolvimento de lactentes nascidos a termo, com peso adequado (AIG) ou pequeno para a idade gestacional (PIG), no $2^{\circ}$ mês de vida. Avaliaramse 67 lactentes: 43 AIG e 24 PIG, utilizando as Bayley Scales of Infant Development. O Index Score (IS) nas Escalas Mental e Motora foi significativamente menor no grupo PIG. Considerando a proporcionalidade corporal (Grupos Controle, Assimétrico e PIG-Simétrico), houve diferença significativa na Escala Motora $(p=0,003)$, com menores pontuações no grupo PIG-Simétrico. Comparados aos percentis de IS do grupo Controle, na Escala Mental, houve diferença entre os grupos Assimétrico X PIG-Simétrico; na Escala Motora, houve diferença entre os grupos Assimétrico X Controle $(p=0,039)$ e PIG-Simétrico X Controle $(p=0,0007)$; não houve diferença entre os grupos Assimétrico e PIG-Simétrico, ambos apresentando menores pontuações que o grupo Controle.
\end{abstract}

PALAVRAS-CHAVE: pequeno para a idade gestacional, desenvolvimento infantil, desnutrição intra-uterina.

\begin{abstract}
Neurodevelopment of full-term small-for-gestacional age infants in the second month of life
ABSTRACT - The objective of the present study was to assess and to compare the neurodevelopment of full-term adequate (AGA) or small-for-gestational age (SGA) infants in the second month of life. Sixty-seven infants were evaluated: 43 AGA and 24 SGA, making use of the Bayley Scales of Infant Development. The SGA group Index Score (IS) was significantly lower in Mental and Motor Scales. Considering the body proportionality (Asymmetric, Symmetric-SGA and Control group) there was difference in Motor Scale $(p=0.003)$ with lower scores in the Symmetric-SGA group. Comparing to the Control group IS percentiles, in Mental Scale there was difference between Asymmetric X Symmetric-SGA; in Motor Scale, there was difference between the Asymmetric X Control $(p=0.039)$ and Symmetric-SGA X Control ( $p=0.007)$ groups; there was no difference between Asymmetric and Symmetric-SGA although both exhibited lower scores than the Control group.
\end{abstract}

KEY WORDS: small-for-gestational age, infant development, intrauterine malnutrition.

O termo restrição do crescimento intra-uterino (RCIU) é freqüentemente mal interpretado em obstetrícia. O recém-nascido (RN) com RCIU é definido como aquele que não atingiu seu crescimento potencial genético intra-uterino, sugerindo um processo patológico durante a vida fetal ${ }^{1}$. Estudos mais recentes mostram a tendência à utilização do termo pequeno para a idade gestacional (PIG) para os fetos ou RN que falharam em atingir o padrão de peso ou antropométrico arbitrário para determinada idade gestacional' ${ }^{1}$. Não obstante, os termos RCIU e PIG são, com freqüência, utilizados como sinônimos e os mesmos limites estatísticos são utilizados para sua identificação. No entanto, cabe destacar a possibilidade de alguns neonatos PIG serem apenas constitucionalmente pequenos, quando analisados segundo seu potencial genético de crescimento, representando a porção final da curva de distribuição normal ${ }^{2}$. O conceito de neonato PIG é, portanto, de definição estatística e a interpretação de seu desenvolvimento deve considerar esse importante fator. Os lactentes nascidos PIG cons-

\footnotetext{
Faculdade de Ciências Médicas/Universidade Estadual de Campinas (UNICAMP), Campinas SP, Brasil: 'Mestranda em Ciências Médicas; ${ }^{2}$ Professor Livre Docente do Departamento de Neurologia e Centro de Investigações em Pediatria (CIPED); ${ }^{3}$ Professor Assistente Doutor do Departamento de Pediatria e CIPED; ${ }^{4}$ Professor Titular do Departamento de Neurologia. Apoio: Pesquisa financiada pela Fundação de Amparo à Pesquisa do Estado de São Paulo, Processo nº 00/07234-7.
}

Recebido 26 Janeiro 2004, recebido na forma final 21 Julho 2004. Aceito 20 Setembro 2004.

Dra. Vanda Maria Gimenes Gonçalves - Departamento de Neurologia/FCMIUnicamp - CP 6111 - 13081-970 Campinas SP - Brasil. E-mail: vandagg@uol.com.br 
tituem alvo de pesquisa por serem considerados modelos de desnutrição em idade mais precoce. Essas crianças podem apresentar maior morbi-mortalidade e taxas de deficiências físicas, neurológicas e mentais mais elevadas, embora haja controvérsias a respeito desses achados ${ }^{3}$. Uma revisão dos estudos sobre o desenvolvimento de crianças com RCIU demonstrou resultados conflitantes entre os diversos autores, atribuídos à heterogeneidade dos lactentes selecionados, aos diferentes instrumentos de avaliação do desenvolvimento e à variedade da duração do acompanhamento ${ }^{4}$. Todavia, há concordância de que o declínio do peso ao nascimento está associado ao aumento linear na taxa de morbidade e ao maior risco de anormalidades leves do neurodesenvolvimento, como alterações cognitivas, dificuldades acadêmicas e problemas comportamentais ${ }^{5}$. Estas condições, resultantes de RCIU, têm numerosas etiologias ${ }^{6}$ e o prognóstico, em longo prazo, depende da etiologia específica e do grau da restrição sofrida ${ }^{7}$, bem como do momento da sua ocorrência durante a gestação ${ }^{8,9}$. Nesse sentido, ao considerar a vulnerabilidade do cérebro em desenvolvimento, conseqüente à restrição do crescimento de causa nutricional, três fatores principais são ressaltados: a gravidade da restrição, o momento (timing) da ocorrência e a duração do evento em relação ao período de crescimento rápido do cérebro ${ }^{10}$.

Na tentativa de descrever o RCIU de forma mais precisa do que a definida pela adequação do peso e idade gestacional, alguns índices de proporcionalidade corporal, como o índice ponderal (que relaciona o peso e a estatura), têm sido utilizados pra referir diferentes dimensões do crescimento fetal ${ }^{11}$. Em teoria, neonatos com padrão de crescimento assimétrico são aqueles com perímetro craniano e estatura normais e peso ao nascimento reduzido; e os neonatos com padrão simétrico são aqueles com perímetro craniano, estatura e peso ao nascimento reduzidos proporcionalmente. A avaliação do desenvolvimento da criança diz respeito principalmente ao desempenho correto de acordo com o tempo maturacional para qualquer comportamento particular. Entretanto, essas etapas evolutivas têm sido objeto de estudos com enfoque psicológico. Sob o aspecto neurológico e pediátrico, não apresentam sistematização e quantificação ${ }^{12}$. O diagnóstico de normalidade do desenvolvimento é altamente complexo, quando se trata de organismo em pleno crescimento, especialmente nos primeiros meses de vida, período caracterizado pela variabilidade do comportamento, do tono muscular, da atividade postural e das habilidades funcionais. As variações sofrem a ação direta da maturação do sistema nervoso e dificultam a interpretação de possíveis anormalidades do desenvolvimento ${ }^{13}$.

Assim, o presente estudo teve por objetivo avaliar e comparar o desenvolvimento de lactentes nascidos com peso adequado (AIG) ou PIG e verificar a relação do peso ao nascimento com a performance no $2^{\circ}$ mês de vida, utilizando as Escalas Bayley de Desenvolvimento Infantil II (BSID-II) ${ }^{14}$.

\section{MÉTODO}

Utilizou-se o corte seccional no $2^{\circ}$ mês, de um estudo prospectivo no $1^{\circ}$ ano de vida, de duas coortes de lactentes nascidos a termo PIG ou AIG.

Foram obedecidos os princípios da Resolução 196 do Conselho Nacional de Saúde e o estudo foi aprovado pelo comitê de ética da Faculdade de Ciências Médicas da Universidade Estadual de Campinas (UNICAMP).

No período de maio de 2000 a julho de 2003, foram selecionados RN a termo, cujos pais ou responsáveis legais assinaram o termo de consentimento livre e esclarecido. Para cada nascimento PIG, foram selecionados os dois nascimentos AIG subsequentes. Foram incluídos: RN do alojamento conjunto da maternidade do Centro de Atenção Integral à Saúde da Mulher (CAISM)/UNICAMP; procedentes da região metropolitana de Campinas; com idade gestacional entre 37 semanas completas e 41 semanas e 6 dias ${ }^{15}$; RN com peso ao nascimento entre o percentil 10 e 90 da curva de crescimento fetal ${ }^{16} \mathrm{com}$ pondo o grupo AIG; RN com peso ao nascimento abaixo do percentil 10 da curva de crescimento fetal ${ }^{16}$ compondo o grupo PIG. Foram excluídos os RN com síndromes genéticas; com grandes malformações; os resultantes de gestação de fetos múltiplos; os que necessitaram de internação em unidade de terapia intensiva neonatal e aqueles com infecção congênita confirmada.

Para avaliar a proporcionalidade corporal ao nascimento utilizou-se o índice ponderal ${ }^{11}$ (IP), calculado segundo a fórmula de Rohrer: Peso (gramas) X $100 /$ Estatura $^{3}$ (cm). Classificou-se como Simétrico, o lactente com IP $\geq 2,32$ e como Assimétrico, o lactente com IP $<2,32^{17}$.

As BSID-II ${ }^{14}$ foram utilizadas como teste padronizado para avaliação do neurodesenvolvimento. A classificação nas escalas de acordo com o Index Score (IS) obedeceu a seguinte pontuação: IS $\geq 115$-performance acelerada; IS entre 85 e 114 - dentro dos limites normais; IS entre 70 e 84 - performance levemente atrasada; IS $\leq 69$ - performance significantemente atrasada.

Classificou-se o desenvolvimento mental e motor segundo a pontuação numérica do IS como: performance adequada quando IS $\geq 85$ (performance acelerada e dentro dos limites normais) e performance inadequada quando IS $<85$ (performance levemente e significantemente atrasada). 
A equipe responsável pela avaliação do neurodesenvolvimento desconhecia os antecedentes neonatais e o peso ao nascimento. Os dados registrados nos roteiros de avaliação foram transcritos e armazenados nos moldes de arquivo para o banco de dados do programa computacional SPSS (Statistical Package for Social Sciences), versão 11.0.

As variáveis foram analisadas por freqüência, média, mediana e desvio-padrão. Aquelas com distribuição normal foram analisadas pelo teste $t$ de Student e as que, após transformação, continuaram sem distribuição normal, foram analisadas pelo teste Mann-Whitney, ambas para amostras independentes. Para analisar médias para 3 grupos independentes utilizou-se o teste KruskalWallis; para análise de freqüências foram feitos teste de $\chi^{2}$ ou Exato de Fisher e o indicador razão de chances pre- valentes $(\mathrm{RCP})$. Considerou-se $5 \%$ o limite de nível de significância $(\alpha=0,05)$ em todos os testes realizados.

\section{RESULTADOS}

Foram selecionados 125 neonatos que preencheram os critérios de inclusão. Compareceram para a avaliação programada do $2^{\circ}$ mês, 74 lactentes; desses, foram avaliados 67 (43 AIG e 24 PIG). Sete lactentes $(9,46 \%)$ do grupo AIG foram excluídos por motivos variados como choro, sono ou outros desconfortos não solucionados durante a avaliação. Um único lactente AIG com avaliação apenas na Escala Mental foi mantido na casuística.

As características clínicas descritivas da amostra estudada foram listadas na Tabela 1. A amostra,

Tabela 1. Distribuição das freqüências das características clínicas ao nascimento.

\begin{tabular}{|c|c|c|c|c|c|c|c|}
\hline \multirow[b]{3}{*}{ Dados do RN } & \multirow[b]{3}{*}{$n$} & \multirow[b]{3}{*}{$(\%)$} & \multicolumn{4}{|c|}{ Grupos } & \multirow[b]{3}{*}{$p$-valor } \\
\hline & & & \multicolumn{2}{|c|}{ AIG } & \multicolumn{2}{|c|}{ PIG } & \\
\hline & & & $f$ & $(\%)$ & $f$ & $(\%)$ & \\
\hline \multicolumn{8}{|l|}{ Sexo } \\
\hline Feminino & 38 & $(56,7)$ & 26 & $(60,5)$ & 12 & $(50,0)$ & $0,407^{(a)}$ \\
\hline Masculino & 29 & $(43,3)$ & 17 & $(39,5)$ & 12 & $(50,0)$ & \\
\hline Total & 67 & & 43 & & 24 & & \\
\hline \multicolumn{8}{|l|}{ Peso (gramas) } \\
\hline$<2.500$ & 15 & $(22,4)$ & 0 & $(0,0)$ & 15 & $(62,5)$ & $<0,001^{(b)}$ \\
\hline$\geq 2.500$ & 52 & $(77,6)$ & 43 & $(100)$ & 9 & $(37,5)$ & \\
\hline Total & 67 & & 43 & & 24 & & \\
\hline \multicolumn{8}{|c|}{ Apgar $1^{\circ}$ minuto $(c)$} \\
\hline$<7$ & 7 & $(11,1)$ & 5 & $(12,5)$ & 2 & $(8,7)$ & $0,494^{(b)}$ \\
\hline$\geq 7$ & 56 & $(88,9)$ & 35 & $(87,5)$ & 21 & (91.3) & \\
\hline Total & 63 & & 40 & & 23 & & \\
\hline \multicolumn{8}{|c|}{ Apgar $5^{\circ}$ minuto } \\
\hline$<7$ & 0 & $(0,0)$ & 0 & $(0,0)$ & 0 & $(0,0)$ & \\
\hline$\geq 7$ & 63 & $(100)$ & 40 & $(100)$ & 23 & $(100)$ & \\
\hline Total & 63 & & 40 & & 23 & & \\
\hline \multicolumn{8}{|l|}{ IG (semanas) } \\
\hline 37 & 3 & $(4,6)$ & 3 & $(7,1)$ & 0 & $(0,0)$ & $0,759^{(b)}$ \\
\hline 38 & 8 & $(12,1)$ & 4 & $(9,5)$ & 4 & $(16,7)$ & \\
\hline 39 & 20 & $(30,3)$ & 14 & $(33,3)$ & 6 & $(25,0)$ & \\
\hline 40 & 28 & $(42,4)$ & 17 & $(40,5)$ & 11 & $(45,8)$ & \\
\hline 41 & 7 & $(10,6)$ & 4 & $(9,5)$ & 3 & $(12,5)$ & \\
\hline Total & 66 & & 42 & & 24 & & \\
\hline \multicolumn{8}{|l|}{ Índice ponderal } \\
\hline Simétrico & 54 & $(80,6)$ & 39 & $(90,7)$ & 15 & $(62,5)$ & $0,007^{(b)}$ \\
\hline Assimétrico & 13 & $(19,4)$ & 4 & $(9,3)$ & 9 & $(37,5)$ & \\
\hline
\end{tabular}

(a) teste $\chi^{2}$; (b) teste exato de Fisher; (c) $\chi^{2}=0,55$ para 2 graus de liberdade $(37+38 ; 39 ; 40+41)$; AIG, adequado para a idade gestacional; PIG,Pequeno para a idade gestacional; IG, idade gestacional; 3 AIG e 1, PIG sem informação de índice de Apgar; 1 AIG sem informação de IG. 
Tabela 2. Características antropométricas ao nascimento.

\begin{tabular}{lcccccccc}
\hline Variáveis & Grupo & $\mathrm{n}$ & média & DP & mínimo & mediana & máximo & $p$-valor \\
\hline Peso (gramas) & PIG & 24 & 2395 & 161 & 2045 & 2445 & 2620 & $<0,001$ \\
& AIG & 43 & 3155 & 296 & 2635 & 3130 & 3850 & \\
\multirow{2}{*}{ Estatura (cm) } & PIG & 24 & 46,9 & 1,65 & 44,0 & 46,5 & 51,0 & $<0,001$ \\
& AIG & 43 & 49,5 & 1,51 & 45,0 & 49,5 & 32,5 & 35,0 \\
PC (cm) & PIG & 24 & 32,4 & 1,14 & 29,5 & 32,5 & 37,0 & $<0,001$ \\
& AIG & 42 & 33,8 & 1,23 & 31,0 & 34,0 & 37,0
\end{tabular}

(a) teste t de Student; n-número de sujeitos; DP, desvio-padrão; AIG, adequado para a idade gestacional; PIG, Pequeno para a idade gestacional; PC, Perímetro craniano; 1 AIG sem informação de medida de PC.

quando distribuída segundo a proporcionalidade corporal ao nascimento, detectou 4 lactentes $(9,3 \%)$ do grupo AIG com padrão de crescimento assimétrico (Tabela 1). A casuística classificada pelo IP constituiu-se de: grupo PIG simétrico (PIG-S, $n=15$, PIG simétrico com IP $\geq 2,32$ ); grupo Assimétrico ( $n$ $=13$, sendo 4 AIG e 9 PIG com IP $<2,32$ ) e grupo Controle $(n=39$, AIG simétrico com IP $\geq 2,32)$. A distribuição dos neonatos quanto à variável sexo foi semelhante nos dois grupos. Houve diferença significativa quanto à distribuição do baixo peso ao nascer, não havendo nenhum neonato AIG com peso ao nascimento inferior a $2500 \mathrm{~g}$. Os grupos não demonstraram risco quanto a sofrimento fetal e neonatal imediato, sendo o índice de Apgar de $5^{\circ}$ minuto $\geq 7$ em $100 \%$. Os grupos apresentaram distribuição homogênea quanto à idade gestacional, em ambos, aproximadamente $70 \%$ nasceram entre a $39^{\mathrm{a}}$ e a $40^{\mathrm{a}}$ semana (Tabela 1 ).
As medidas de peso, estatura e perímetro craniano ao nascimento foram significativamente menores no grupo PIG (Tabela 2). Na avaliação do desenvolvimento, não houve diferença nas performances mental e motora entre os grupos formados pela adequação peso/idade gestacional (PIG e AIG) ou pela proporcionalidade corporal (PIG-S, Assimétrico e Controle) (Tabela 3). Comparando-se os grupos PIG e AIG quanto às pontuações do IS, observouse diferença significativa nas Escalas Mental e Motora, com pontuações menores no grupo PIG (Tabela 4). Comparando-se as pontuações do IS na Escala Mental, nos grupos formados pela proporcionalidade corporal, embora sem diferença estatisticamente significativa, o grupo PIG-S apresentou as menores pontuações. Na Escala Motora a diferença entre os grupos foi significativa, com as menores pontuações no grupo PIG-S (Tabela 4).

Tabela 3. Performance nas Escalas Mental e Motora nas BSID-II no $2^{\circ}$ mês.

\begin{tabular}{|c|c|c|c|c|c|c|c|c|}
\hline \multirow[b]{3}{*}{ Escala } & \multirow[b]{3}{*}{ Grupos } & \multirow[b]{3}{*}{ (n) } & \multicolumn{4}{|c|}{ Performance } & \multirow[b]{3}{*}{$\mathrm{RCP}$} & \multirow[b]{3}{*}{ IC 95\% } \\
\hline & & & \multicolumn{2}{|c|}{ Inadequada } & \multicolumn{2}{|c|}{ Adequada } & & \\
\hline & & & $f$ & $\%$ & $f$ & $\%$ & & \\
\hline \multirow[t]{5}{*}{ Mental } & PIG & (24) & 8 & $(33,3)$ & 16 & $(66,7)$ & 1,29 & {$[0,38-4,33]$} \\
\hline & AIG & (43) & 12 & $(27,9)$ & 31 & $(72,1)$ & 1 & \\
\hline & Assimétrico & (13) & 2 & $(15,4)$ & 11 & $(84,6)$ & 0,46 & {$[0,06-2,84]$} \\
\hline & PIG-S & (15) & 7 & $(46,7)$ & 8 & $(53,3)$ & 2,23 & {$[0,55-9,10]$} \\
\hline & Controle & (39) & 11 & $(28,2)$ & 28 & $(71,8)$ & 1 & \\
\hline \multirow[t]{5}{*}{ Motora } & PIG & (24) & 4 & $(16,7)$ & 20 & $(83,3)$ & 1,90 & {$[0,35-10,42]$} \\
\hline & AIG & (42) & 4 & $(9,5)$ & 38 & $(90,5)$ & 1 & \\
\hline & Assimétrico & (13) & 2 & $(15,4)$ & 11 & $(84,6)$ & 2,12 & {$[0,21-19,04]$} \\
\hline & PIG-S & (15) & 3 & $(20,0)$ & 12 & $(20,0)$ & 2,92 & {$[0,39-21,89]$} \\
\hline & Controle & (38) & 3 & $(7,9)$ & 35 & $(92,1)$ & 1 & \\
\hline
\end{tabular}

n, número de sujeitos; $f$, freqüência observada; RCP, razão de chances prevalentes; IC, intervalo de confiança; PIG, pequeno para a idade gestacional; AIG, adequado para a idade gestacional; S, simétrico; 1 AIG do grupo Controle sem avaliação na Escala Motora. 
Tabela 4. Index Score nas Escalas Mental e Motora no $2^{\circ}$ mês.

\begin{tabular}{llccccc}
\hline Escala & Grupo & $\mathrm{n}$ & Média & DP & IC 95\% & $p$-valor \\
\hline Mental & PIG & 24 & 86,3 & 10,66 & {$[82-90]$} & $0,047^{\text {(a) }}$ \\
& AIG & 43 & 91,4 & 9,75 & {$[88-94]$} & \\
& Assimétrico & 13 & 90,5 & 7,38 & {$[86-95]$} & $0,073^{(\mathrm{c})}$ \\
& PIG-S & 15 & 84,1 & 10,80 & {$[78-90]$} & \\
& Controle & 39 & 91,3 & 10,06 & {$[88-94]$} & \\
Motora & PIG & 24 & 89,5 & 6,11 & {$[87-92]$} & $0,005^{(\mathrm{b})}$ \\
& AIG & 42 & 93,5 & 7,58 & {$[91-96]$} & \\
& Assimétrico & 13 & 89,7 & 7,08 & {$[85-94]$} & $0,003^{(\mathrm{c})}$ \\
& PIG-S & 15 & 88,8 & 6,88 & {$[84-92]$} & \\
& Controle & & & \\
& & 38 & 94,4 & 6,75 & {$[92-96]$} & \\
\hline
\end{tabular}

(a) teste t de Student; (b) teste Mann-Whitney; (c) teste Kruskal-Wallis; n-número de sujeitos; DP, desvio-padrão; IC, Intervalo de confiança; PIG, Pequeno para a idade gestacional; AIG, adequado para a idade gestacional; 1 AIG do grupo Controle sem avaliação na Escala Motora.

A distribuiç̧ão do IS na Escala Mental dos grupos PIG-S, Assimétrico e Controle, ilustrada na Figura 1, demonstrou que $75 \%$ dos PIG-S apresentaram valores abaixo da mediana do grupo Controle. A distribuição do IS da Escala Motora dos grupos segundo a proporcionalidade corporal (Fig 2), demonstrou que aproximadamente $75 \%$ dos lactentes PIG-S apresentaram valores de IS equivalentes aos valores $25 \%$ mais baixos do grupo Controle, ou sejam, equivalentes ao I quartil. Na distribuição do
IS do grupo Assimétrico, $50 \%$ dos casos tiveram o mesmo comportamento. A apresentação tornou visível a diferença na distribuição da pontuação do grupo PIG-S em relação ao Controle.

Considerando-se estes resultados, julgou-se procedente classificar a amostra em relação aos quartis determinados pelo IS do grupo Controle (I, II, III e IV), calculando-se seus percentis 25,50 e 75 . Os valores de percentil do IS do grupo Controle encontrados nas Escalas Mental e Motora, foram res-

Tabela 5. Distribuição do Index score nos quartis estabelecidos pelo grupo Controle nas Escalas Mental e Motora.

\begin{tabular}{|c|c|c|c|c|c|c|c|c|}
\hline \multirow[b]{3}{*}{ Escalas } & \multicolumn{6}{|c|}{ Grupos } & \multirow{2}{*}{\multicolumn{2}{|c|}{$\begin{array}{c}\text { Total } \\
\mathrm{n}=67\end{array}$}} \\
\hline & \multicolumn{2}{|c|}{$\begin{array}{l}\text { Controle } \\
n=39\end{array}$} & \multicolumn{2}{|c|}{$\begin{array}{c}\text { Assimétrico } \\
n=13\end{array}$} & \multicolumn{2}{|c|}{$\begin{array}{l}\text { PIG-S } \\
n=15\end{array}$} & & \\
\hline & $f$ & $(\%)$ & $f$ & $(\%)$ & $f$ & $(\%)$ & $f$ & $(\%)$ \\
\hline \multicolumn{9}{|l|}{ Mental } \\
\hline \multicolumn{9}{|c|}{ Quartil } \\
\hline I & 11 & $(28,2)$ & 2 & $(15,4)$ & 8 & $(53,3)$ & 21 & $(31,3)$ \\
\hline II & 12 & $(30,8)$ & 6 & $(46,1)$ & 5 & $(33,3)$ & 23 & $(34,3)$ \\
\hline III & 9 & $(23,1)$ & 4 & $(30,8)$ & 1 & $(6,7)$ & 14 & $(20,9)$ \\
\hline IV & 7 & $(17,9)$ & 1 & $(7,7)$ & 1 & $(6,7)$ & 9 & $(13,4)$ \\
\hline \multicolumn{9}{|c|}{ Motora } \\
\hline \multicolumn{9}{|c|}{ Quartil } \\
\hline 1 & 11 & $(28,9)$ & 8 & $(61,5)$ & 12 & $(80,0)$ & 31 & $(47.0)$ \\
\hline II & 10 & $(26,3)$ & 2 & $(15,4)$ & 1 & $(6,7)$ & 13 & (19.7) \\
\hline III & 11 & $(28,9)$ & 3 & $(23,1)$ & 1 & $(6,7)$ & 15 & $(22,7)$ \\
\hline IV & 6 & $(15,8)$ & 0 & $(0,0)$ & 1 & $(6,7)$ & 7 & $(10,6)$ \\
\hline
\end{tabular}

$\mathrm{N}$, número de sujeitos; $f$, freqüência observada; PIG, S-pequeno para a idade gestacional simétrico; Escala Mental (I; II-IV): Controle X Assimétrico (teste exato de Fisher $\mathrm{p}=0,298)$; Controle X PIG-S $\left(\chi^{2}\right.$ corrigido $=2,00$; 1 grau de liberdade; $p=0,15$ ); Assimétrico X PIG-S (teste exato de Fisher $p=0,043$ ) Escala Motora (I; II-IV): Controle X Assimétrico (teste exato de Fisher $\mathrm{p}=0,039$ ); Controle XPIG-S $\left(\chi^{2}\right.$ corrigido $=9,43 ; 1$ grau de liberdade; $\mathrm{p}=0,002)$; Assimétrico X PIG-S (teste exato de Fisher $\mathrm{p}=0,255)$; 1 AIG sem avaliação na Escala Motora. 


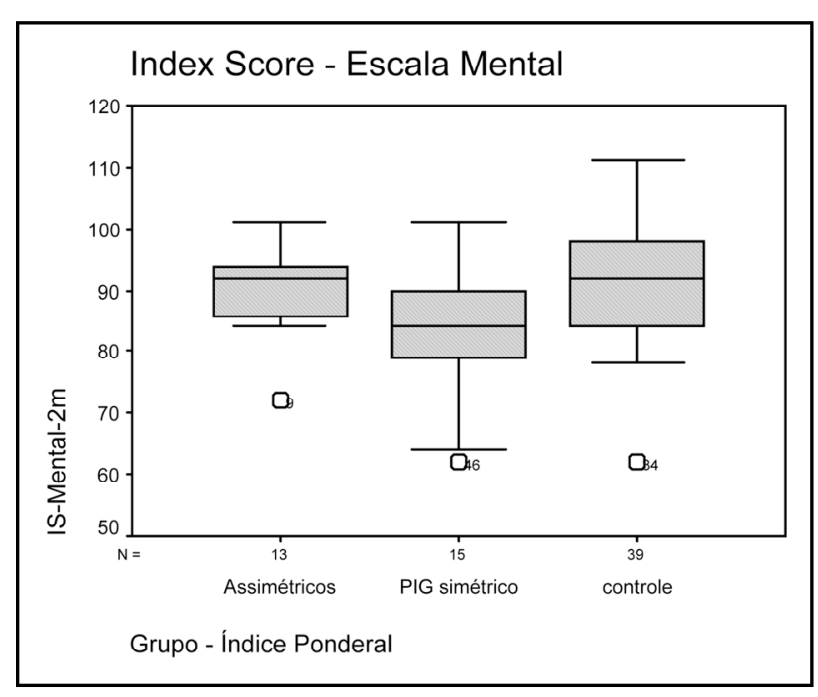

Fig 1. Box-plot da distribuição do Index Score na Escala Mental.

pectivamente: 84 e 90 (percentil 25); 92 e 93 (percentil 50) e 98 e 99 (percentil 75). Para análise desses resultados, comparou-se a freqüência encontrada no I quartil (abaixo do percentil 25) com a encontrada nos demais quartis agrupados (II, III e IV). Verificou-se que, na Escala Mental, mais da metade $(53,3 \%)$ dos PIG-S encontravam-se abaixo do percentil 25 e $46,7 \%$ acima do mesmo, enquanto apenas $15,5 \%$ dos Assimétricos encontraram-se abaixo, e $84,6 \%$ acima do percentil 25. A distribuição do grupo Controle $(28,2 \%$ abaixo e $71,8 \%$ acima do percentil 25) foi semelhante ao grupo Assimétrico (Tabela 5).

Na Escala Motora, os grupos PIG-S e Assimétrico apresentaram comportamentos semelhantes, sendo que $80,0 \%$ e $61,5 \%$, respectivamente, encontraramse abaixo do percentil 25 . No entanto, diferiram do grupo Controle em que apenas $28,9 \%$ se encontraram abaixo do percentil 25 (Tabela 5).

\section{DISCUSSÃO}

As vulnerabilidades potenciais do desenvolvimento do cérebro da criança são amplamente reconhecidas; no entanto, relativamente pouco se conhece a respeito dos mecanismos envolvidos. Os resultados de experimentos em animais podem ser estendidos apenas cautelosamente aos seres humanos, mas alguns desses achados certamente têm implicações clínicas importantes. As teorias contemporâneas enfatizam o potencial de autoorganização das estruturas cerebrais, particularmente das regiões envolvidas no armazenamento de informações, qual seja, a plasticidade em resposta a experiências ${ }^{18}$. A preocupação com a mor-

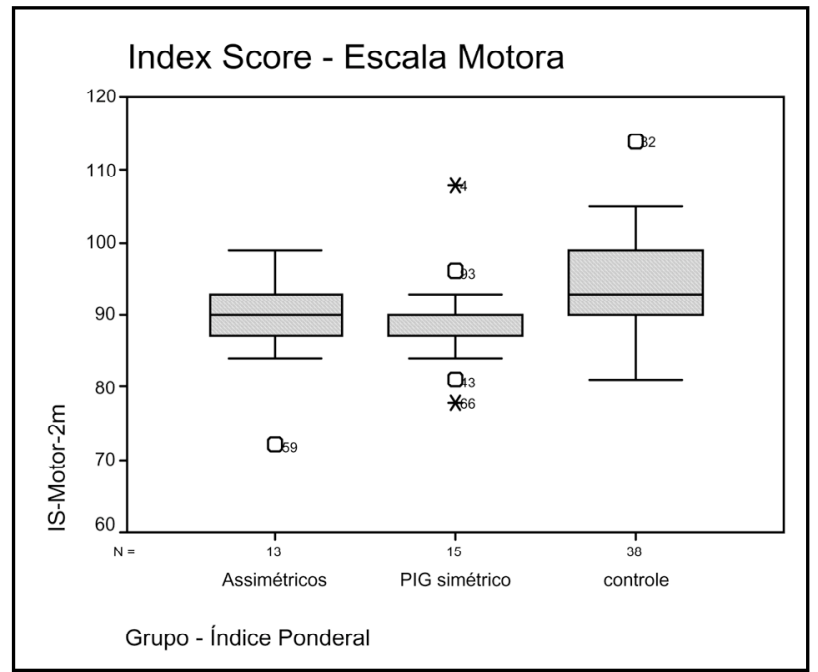

Fig 2. Box-plot da distribuição do Index Score na Escala Motora.

bidade neurológica associada aos lactentes PIG é atribuída aos experimentos em animais das décadas de 1960 e 70 cujos resultados demonstravam a existência de um período crítico ${ }^{19}$ no desenvolvimento do sistema nervoso, em que a desnutrição em idade precoce poderia causar danos irreversíveis tanto no número de células neurais como nos processos de mielinização e sinaptogênese ${ }^{20-22}$.

Na casuística apresentada, os grupos PIG e AIG não demonstraram diferenças quando analisados quanto à performance mental e motora; no entanto, considerando a pontuação do IS, o grupo PIG apresentou resultados significativamente menores nas duas Escalas. O significado clínico desse achado sugere que os lactentes nascidos PIG, embora estejam no intervalo de normalidade do desenvolvimento, possam estar em maior risco de resultados adversos, podendo ser detectados já no $2^{\circ}$ mês de vida. Dessa forma, presumiu-se que esse achado sugira a ocorrência no grupo AIG, e ainda não manifesta no grupo PIG, da assim chamada transformação maior da função neural descrita por Precht $\left.\right|^{23}$. É referido que, no lactente normal, aproximadamente ao final do $2^{\circ}$ mês de vida, muitas funções mudam para uma condição mais adaptativa do que a observada durante esses primeiros dois meses $^{23,24}$.

A diferença no $2^{\circ}$ mês de vida de lactentes PIG foi observada utilizando uma escala de avaliação modificada, reunindo provas de diferentes autores, e que se acentuou nas avaliações do $6^{\circ}$ e $12^{\circ}$ meses $^{8}$. Houve correlação positiva com as mudanças nos padrões de peso e de medidas do perímetro craniano 
e mudanças nas pontuações do neurodesenvolvimento do nascimento até o $6^{\circ}$ mês de vida.

Na casuística da pesquisa atual, comparandose os grupos classificados de acordo com a proporcionalidade corporal, observaram-se as menores pontuações nas Escalas Mental e Motora no grupo PIG com padrão de crescimento simétrico. Esse resultado sugere o maior comprometimento conseqüente a maior gravidade da restrição do crescimento e à maior duração do insulto, possivelmente durante todo o período pré-natal.

São atribuídas diferentes etiologias para a RCIU. Considera-se que o padrão de crescimento assimétrico seja conseqüente à insuficiência placentária, em geral limitada ao final da gestação, enquanto o crescimento simétrico pode ser resultante de crescimento fetal potencial reduzido devido a causas genéticas ou a influências ambientais prolongadas durante a gestação ${ }^{25}$. Acredita-se que o crescimento do cérebro seja poupado na insuficiência placentária tardia, devido à brevidade do insulto26. Assim, justificam-se os resultados encontradosnesta casuística. Todavia, há controvérsias entre os autores que consideram o neurodesenvolvimento de acordo com o índice ponderal de crianças nascidas PIG.

Avaliando o desenvolvimento de lactentes nascidos a termo com RCIU, divididos de acordo com o índice ponderal baixo ou adequado e comparando ao grupo controle, não foi encontrada diferença aos 6 e 15 meses de idade ${ }^{9}$. Entretanto, aos 24 meses o grupo com RCIU simétrico mostrou, embora sem significado estatístico, as menores pontuações na performance mental. Com três anos de idade, esse grupo apresentou as pontuações menores nos testes de avaliação de percepção, na resolução de problemas, em testes de avaliação da memória e de linguagem. Os autores consideraram apropriado utilizar a medida da estatura como complemento do peso de nascimento, para a avaliação clínica do crescimento e do desenvolvimento de lactentes com RCIU.

$\mathrm{Na}$ avaliação de lactentes até o $2^{\circ}$ ano de vida, utilizando um teste de triagem de desenvolvimento modificado ${ }^{7}$, foi observado que os lactentes PIG apresentaram freqüência significativamente maior de anormalidades do desenvolvimento; entretanto, sem associação com a proporcionalidade corporal. Resultados diferentes foram observados aos 13 meses de idade, comparando lactentes PIG com um grupo controle ${ }^{27}$, utilizando as BSID-II; apenas na
Escala Mental a pontuação foi significativamente mais baixa nos lactentes PIG, não havendo diferenças nas habilidades motoras. $O$ desenvolvimento mental do lactente foi positivamente associado ao índice ponderal ao nascimento e à escolaridade dos pais. Entretanto, os autores referiram a pouca confiabilidade das Escalas Bayley, bem como de outros instrumentos similares, em predizer nessa faixa etária, o potencial desenvolvimento posterior.

Mais recentemente foram relacionados o padrão de crescimento fetal no terceiro trimestre de gestação e o neurodesenvolvimento com um ano de idade, utilizando o exame neurológico e avaliação do desenvolvimento ${ }^{28}$. Embora a incidência de anormalidades fosse bastante alta, em aproximadamente $1 / 3$ dos lactentes, não foi encontrada diferença entre os fetos que já eram pequenos e fetos com RCIU no $3^{\circ}$ trimestre de gestação. Os autores interpretaram que os sinais neurológicos, embora sutis e representando alterações neurológicas menores, poderiam predizer problemas escolares mais tardios.

Com o objetivo de conhecer a influência de algumas variáveis (lactentes brasileiros normais, avaliados pelos mesmos profissionais, utilizando o mesmo instrumento) e analisar mais detalhadamente os resultados da casuística da pesquisa atual, considerou-se interessante determinar os percentis 25, 50 e 75 do IS do grupo Controle. Esse recurso foi, portanto, utilizado para a comparação de grupos com características populacionais semelhantes. Os valores medianos encontrados estiveram abaixo da média teórica da escala (média $=100$ ), referida no manual das BSID-II ${ }^{14}$. No grupo Controle, a mediana (percentil 50) na escala Mental foi 92 e na escala Motora foi 93. Mesmo considerando esses menores valores de mediana, observou-se que, na Escala Mental houve diferença entre os grupos Assimétrico e PIG-S. Entretanto, os dois grupos não apresentaram diferenças em relação ao grupo Controle.

A Escala Motora diferenciou o grupo Controle do grupo Assimétrico, e com maior evidência, do grupo PIG-S. Confirmaram-se as menores pontuações dos lactentes PIG-S e Assimétrico. No entanto, a diferença não foi significativa entre esses últimos. Na Escala Motora, os lactentes Assimétricos apresentaram distribuição do IS similar ao grupo PIG-S.

Concluindo, esse estudo mostrou que lactentes nascidos PIG e AIG se diferenciaram na distribuição do IS nas Escalas Mental e Motora, com menores 
pontuações do grupo PIG, embora não tenham apresentado diferenças quanto ao intervalo de normalidade. Os grupos formados pela proporcionalidade corporal se diferenciaram na Escala Motora, com as menores pontuações obtidas pelo grupo PIG com padrão de crescimento simétrico. Calculada a mediana do grupo Controle, os grupos Assimétrico e PIG-S se diferenciaram na Escala Mental; na Escala Motora, houve diferença entre os grupos Assimétrico e Controle e entre PIG-S e Controle, não havendo diferença entre os grupos Assimétrico e PIG-S.

\section{REFERÊNCIAS}

1. Goldenberg RL, Cliver SP. Small for gestational age and intrauterine g rowth restriction: definitions and standards. Clin Obstet Gynecol 1997;40:704-714.

2. Mamelle M, Cochet V, Claris O. Definition of fetal growth restriction according to constitutional growth potential. Biol Neonate 2001;80:277285.

3. Strauss RS. Adult functional outcome of those born small for gestational age: twenty-six-year follow-up of the 1970 British Birth Cohort. JAMA 2000;283:625-632.

4. Bos AF, Einspieler C, Prechtl HFR. Intrauterine growth retardation, general movements, and neurodevelopmental outcome: a review. Dev Med Child Neurol 2001;43:61-68.

5. Zubrick SR, Kurinczuk JJ, Mcdermott BMC, Mckelvey RS, Silburn SR, Davies LS. Fetal growth and subsequent mental health problems in children aged 4 to 13 years. Dev Med Child Neurol 2000;42:14-20.

6. Kramer MS. Intrauterine growth and gestational duration determinants. Pediatrics 1987;4:502-511.

7. Tenovuo A, Kero P, Korvenranta H. Developmental outcome of 519 small-for-gestational age children at the age of two years. Neuropediatrics 1988;19:41-45.

8. Ounsted M, Moar VA, Scott A. Neurological development of small-forgestational age during the first year of life. Early Hum Develop 1988;16:163-172.

9. Villar J, Smeriglio V, Martorell R, Brown CH, Klein RE. Heterogeneous growth-retarded infants during the first 3 years of life. Pediatrics 1984;74:783-791.

10. Dobbing J, Sands J. Head circumference, biparietal diameter and brain growth in fetal and postnatal life. Early Hum Develop 1978;2:81-87.
11. Miller HC, Hassanein K. Diagnosis of impaired fetal growth in newborn infants. Pediatrics 1971;48:511-523.

12. Diament $\mathrm{A}$. Introdução à semiologia neurológica infantil. In Diament $\mathrm{A}$, Cypel S. (EDS) Neurologia Infantil. 3. Ed. São Paulo: Atheneu; 1996:3-5.

13. Souza RCT. Vigilância neuromotora de lactentes acometidos por indicadores de risco para asfixia perinatal no primeiro semestre de vida. Dissertação de Mestrado, Universidade Estadual de Campinas. Campinas, 1998.

14. Bayley N. Bayley Scales of Infant Development II, 2nd Edition. San Antonio: The American Psychological Corporation, Harcourt Brace \& Company, 1993:2-30.

15. Organização Mundial de Saúde, CID-10. Classificação Estatística Internacional de Doenças e Problemas Relacionados à Saúde, $10^{\mathrm{a}}$ revisão, v. 1. Tradução do Centro Colaborador da OMS para a Classificação de Doenças em Português. Definições, $7^{a}$ edição. São Paulo: Editora da Universidade São Paulo, 1999:1181-1186.

16. Battaglia FC, Lubchenco LO. A practical classification of newborn infants by weight and gestational age. J Pediat 1967;71:159-163.

17. Lockwood CJ, Weiner S. Assessment of fetal growth. Clin Perinatol 1986;13:3-35

18. Black JE. How a child builds its brain: some lessons from animal studies of neural plasticity. Prev Med 1998;27:168-171.

19. Goodman CS, Shatz CJ. Developmental mechanisms that generate precise patterns of neuronal connectivity. Cell 72 / Neuron 1993;10:(Suppl):S77-S98.

20. Dobbing J. Undernutrition and the developing brain: the relevance of animal models to the human problem. Am J Dis Child 1970; 120: 411-415.

21. Dobbing J, Sands J. Vulnerability of developing brain: IX. The effect of nutritional growth reta rdation on the timing of the brain growth-spurt. Biol Neonate 1971;19:363-378.

22. Winick M. Nutrition, growth and mental development: biological correlation. Am J Dis Child 1970;120:416-418.

23. P rechtl HFR. Continuity of neural functions from prenatal to postnatal life. Clin Dev Med, 94. London: Mac Keith Press, 1984;1-50.

24. Prechtl HFR. New perspectives in early human development. Eur J Obstet Gynecol Reprod Biol 1986;21:347-355.

25. Villar J, Belizán JM. The relative contribution of prematurity and fetal growth retardation to low birth weight in developing and developed societies. Am J Obstet Gynecol 1982;143:793-798.

26. Villar J, Belizán JM. The timing factor in the pathophysiology of the intrauterine growth retardation syndrome. Obstet Gynecol Surv 1982;37:499-506.

27. Markestad T, Vik T, Ahlsten G, et al. Small-for-gestational-age (SGA) infants born at term: growth and development during the first year of life. Acta Obstet Gynecol Scand 1997;76:(Suppl 195)S93-S101.

28. Roth S, Chang TC, Robson S, Spencer JA, Wyatt JS, Stewart AL. The neurodevelopmental outcome of term infants with different intrauterine growth characteristics. Early Hum Dev 1999;55:39-50. 